\title{
Pulmonary Tumor Thrombotic Microangiopathy Induced by Ureteral Carcinoma: A Necropsy Case Report
}

\author{
Satoshi Marumo ${ }^{\mathrm{a}, \mathrm{b}} \quad$ Masahiro Sakaguchi $^{\mathrm{b}}$ Takashi Teranishi $^{\mathrm{b}}$ \\ Yuichi Higami $^{\mathrm{b}}$ Yoshiyuki Koshimo $^{\mathrm{b}}$ Motokazu Kato $^{\mathrm{b}}$ \\ ${ }^{a}$ Respiratory Disease Center, Tazuke Kofukai Medical Research Institute, Kitano Hospital, \\ and ${ }^{\mathrm{b}}$ Department of Respiratory Medicine, Kishiwada City Hospital, Osaka, Japan
}

\section{Key Words}

Pulmonary tumor thrombotic microangiopathy - Ureter carcinoma - Pulmonary hypertension . Respiratory failure

\begin{abstract}
Background: Pulmonary tumor thrombotic microangiopathy (PTTM) is a fatal cancer-related pulmonary complication with rapidly progressing dyspnea and pulmonary hypertension that occasionally induces sudden death. We report the first case of PTTM induced by ureter carcinoma. Case Presentation: The patient was an 80-year-old Japanese female with chief complaints of dry cough and dyspnea. An echocardiogram revealed severe pulmonary hypertension. A chest radiograph showed ground glass opacity of bilateral lower lung field predominance, and an abdominal computed tomography scan revealed a left ureter mass suggestive of ureter carcinoma. The patient died of respiratory failure on the eighth day of hospitalization. Postmortem examination indicated that the primary lesion was a left ureter cancer with tumor microemboli extending to both lungs through the right side of the heart. The final diagnosis of this case was PTTM induced by ureter carcinoma. Conclusion: The pathogenesis and pathophysiology of PTTM remains obscure with no effective management available. In cases of rapidly progressing respiratory failure with pulmonary hypertension, it is necessary to consider PTTM in the differential diagnosis.


Marumo et al.: Pulmonary Tumor Thrombotic Microangiopathy Induced by Ureteral Carcinoma: A Necropsy Case Report

\section{Background}

Pulmonary tumor thrombotic microangiopathy (PTTM) is a fatal cancer-related pulmonary complication and was first described by von Herbay et al. [1] in 1990. It is characterized by rapidly progressing dyspnea and pulmonary hypertension and occasionally induces sudden death [2]. Usually, PTTM is associated with adenocarcinoma, particularly gastric adenocarcinomas and adenocarcinomas of the pancreas, breast, and liver. Here, we present a rare case of PTTM induced by ureter carcinoma, which has not been reported to date.

\section{Case Presentation}

An 80-year-old Japanese female presented with a 1-month history of progressive dyspnea and dry cough. On admission, she had tachycardia (102 beats per minute), tachypnea (32 respirations per minute), and normotensive blood pressure (123/75 mm $\mathrm{Hg}$ ). Initial oxygen saturation was $83 \%$ with the patient breathing room air. Blood tests revealed a high C-reactive protein level $(201.4 \mathrm{mg} / \mathrm{l})$ and a white blood cell count of $18,700 / \mu \mathrm{l}$. A chest radiograph showed bilateral opacities (fig. 1a), and a chest high-resolution computed tomography (HRCT) scan revealed ground glass opacity (GGO) of bilateral peripheral lung field predominance (fig. 1b). Written informed consent was obtained from the patient for the publication of this case report and any accompanying images. A copy of the written consent is available for review from the editor of this journal.

The patient was first treated with intravenous antibiotics based on an initial diagnosis of bacterial pneumonia. Despite this treatment, her condition worsened dramatically over the following days. A transthoracic echocardiogram showed a normal ejection fraction with an extremely elevated estimated systolic pulmonary artery pressure of $114 \mathrm{~mm} \mathrm{Hg}$. Plasma coagulation tests indicated activation of coagulation systems with D-dimers elevated to $9,700 \mathrm{mg} / \mathrm{l}$ (compared to a normal level of $<1,000 \mathrm{mg} / \mathrm{l}$ ). Although pulmonary thromboembolic disease was suspected, CT pulmonary angiography showed no evidence of pulmonary emboli but revealed a left ureteral nodule involving the left common iliac vein suggestive of ureteral carcinoma (fig. 1c). We then considered the diagnosis of PTTM induced by ureteral carcinoma. The patient refused chemotherapy and selected palliative care for the suspected ureteral carcinoma. She died of respiratory failure on the eighth day of hospitalization.

Postmortem examination revealed tumor cells in the ureter of the left kidney positive for cytokeratin 7 and cytokeratin 20 upon immunohistochemical analysis, which led to the pathological diagnosis of urothelial carcinoma (fig. 2a, b). Tumor cells morphologically similar to the carcinoma of the ureter were identified in the pulmonary arterioles. Marked intimal fibrocellular proliferation and fibrin thrombi were also observed in the pulmonary arterioles (fig. 2c, d). In some arterioles, recanalization of organized thrombi was identified. Extravasation of tumor cells and carcinomatous lymphangitis were not seen. These findings are consistent with the histopathologic features of PTTM. The final diagnosis was PTTM induced by ureteral carcinoma.

\section{Discussion}

First described by von Herbay et al. [1] in 1990, PTTM is a fatal cancer-related pulmonary complication, which is characterized by rapidly progressing dyspnea and pulmonary hypertension and occasionally induces sudden death [2]. PTTM is observed in $0.8-3.3 \%$ of 
Marumo et al.: Pulmonary Tumor Thrombotic Microangiopathy Induced by Ureteral Carcinoma: A Necropsy Case Report

autopsies of patients with malignant tumors [1] and usually it is associated with adenocarcinoma, particularly gastric adenocarcinomas and adenocarcinomas of the pancreas, breast, and liver. PTTM is further characterized by pathological findings of widespread fibrocellular intimal proliferation of small pulmonary arteries and arterioles in patients with metastatic carcinoma. Tumor cell emboli in PTTM may also activate both local and systemic coagulation systems, leading to thrombosis, fibromuscular intimal proliferation, luminal stenosis, and finally occlusion [1].

In the present case, there are four reasons why the primary lesion was diagnosed to be a left ureteral nodule based only on the specimen from the left kidney. First, a whole body CT scan revealed a nodular lesion only at the left ureter. Second, immunohistochemical staining revealed both cytokeratin 7 - and cytokeratin 20-positive carcinoma, indicating urothelial carcinoma. Third, urothelial carcinoma is reported to cause retrograde metastasis to the ureter of the ipsilateral kidney [3]. Finally, the serum concentration of CYFRA in the present case was elevated to $76.8 \mathrm{ng} / \mathrm{ml}$ (compared to a normal level of $<3.5 \mathrm{ng} / \mathrm{ml}$ ). CYFRA is usually considered a lung cancer-specific tumor marker, but Suyama et al. [4] have reported that CYFRA is also a useful tumor marker for urothelial carcinoma. Therefore, we conclude that the primary lesion was a left urothelial carcinoma that invaded the inferior vena cava with tumor microemboli extending to both lungs through the right side of the heart.

In the present case, PTTM was confirmed by both clinical and pathological findings. A transthoracic echocardiogram showed a normal ejection fraction with an extremely elevated estimated systolic pulmonary artery pressure of $114 \mathrm{~mm} \mathrm{Hg}$. There was no evidence of pulmonary emboli by CT pulmonary angiography, ruling out a diagnosis of pulmonary thromboembolic disease. After hospital admission, the patient's dyspnea progressed rapidly and she died of respiratory failure on the eighth day of hospitalization. PTTM typically presents with clinically severe manifestations, such as rapidly progressing dyspnea, pulmonary hypertension, and right-side heart failure, and often induces sudden death [1]. The clinical manifestations in the present case were consistent with PTTM. Marked intimal fibrocellular proliferation and fibrin thrombi were also observed in the pulmonary arterioles. In some arterioles, recanalization of organized thrombi was identified. Extravasation of tumor cells and carcinomatous lymphangitis were not seen. These findings were consistent with the histopathologic features of PTTM.

The radiologic diagnosis of pulmonary tumor embolism is difficult because the physical evidence may be minimal or nonspecific [5], including peripheral wedge-shaped opacities, multifocal dilatation or beading of vessels, diffuse thickening of the interlobular septa (lymphangitic carcinomatosis), and enlarged central arteries. Unfortunately, the clinical or radiological diagnosis of tumor emboli in patients with extrathoracic malignancies is not often established before death. In the present case, a chest HRCT scan revealed GGO of bilateral peripheral lung field predominance. GGO is reported to occur with small-vessel occlusive diseases such as pulmonary microembolism and acute chest syndrome in sickle cell anemia [6]. Furthermore, experimental pulmonary infarction in a pig model has been reported to show GGO on HRCT [7]. Bilateral GGO may be a nonspecific finding on HRCT but PTTM must be suspected when it presents in patients with malignant tumors.

\section{Conclusions}

We present the first case of PTTM induced by ureteral carcinoma. PTTM is usually associated with adenocarcinoma, particularly gastric adenocarcinomas and adenocarcinomas of the pancreas, breast, and liver. Since lung biopsy is essential for the definitive diagnosis of 


\begin{tabular}{l|l}
\hline DOI: $10.1159 / 000366474$ & $\begin{array}{l}\text { (c) 2014 S. Karger AG, Basel } \\
\text { www.karger.com/cro }\end{array}$ \\
\hline \multicolumn{2}{l}{ Marumo et al.: Pulmonary Tumor Thrombotic Microangiopathy Induced by Ureteral }
\end{tabular}

Carcinoma: A Necropsy Case Report

PTTM, PTTM is difficult to diagnose before death. Therefore, to decrease the underdiagnosis

of and mortality as a result of PTTM, PTTM should always be added to the differential diagnosis of acute respiratory failure in patients with carcinoma.

\section{Disclosure Statement}

The authors declare that they have no competing interests.

\section{References}

1 von Herbay A, Illes A, Waldherr R, Otto HF: Pulmonary tumor thrombotic microangiopathy with pulmonary hypertension. Cancer 1990;66:587-592.

2 Hibbert M, Braude S: Tumour microembolism presenting as 'primary pulmonary hypertension'. Thorax 1997;52:1016-1017.

-3 Habuchi T: Origin of multifocal carcinomas of the bladder and upper urinary tract: molecular analysis and clinical implications. Int J Urol 2005;12:709-716.

-4 Suyama T, Nakajima K, Kanbe S, Tanaka N, Hara H, Ishii N: Prognostic significance of preoperative serum CYFRA 21-1 in patients with upper urinary tract urothelial carcinoma. Int J Urol 2011;18:43-47.

-5 Han D, Lee KS, Franquet T, Muller NL, Kim TS, Kim H, Kwon OJ, Byun HS: Thrombotic and nonthrombotic pulmonary arterial embolism: spectrum of imaging findings. Radiographics 2003;23:1521-1539.

6 Bhalla M, Abboud MR, McLoud TC, Shepard JA, Munden MM, Jackson SM, Beaty JR, Laver JH: Acute chest syndrome in sickle cell disease: CT evidence of microvascular occlusion. Radiology 1993;187:45-49.

7 Horvath G, Noma S, Moskowitz GW, Khan A, Balazs G, Herman PG: Experimental pulmonary infarction in a pig model. Invest Radiol 1992;27:829-835. 


\section{Case Reports in Oncology}

\begin{tabular}{l|l}
\hline \multicolumn{2}{l}{ Case Rep Oncol 2014;7:605-610 } \\
\hline DOI: 10.1159/000366474 & $\begin{array}{l}\text { C 2014 S. Karger AG, Basel } \\
\text { www.karger.com/cro }\end{array}$ \\
\hline
\end{tabular}

Marumo et al.: Pulmonary Tumor Thrombotic Microangiopathy Induced by Ureteral Carcinoma: A Necropsy Case Report
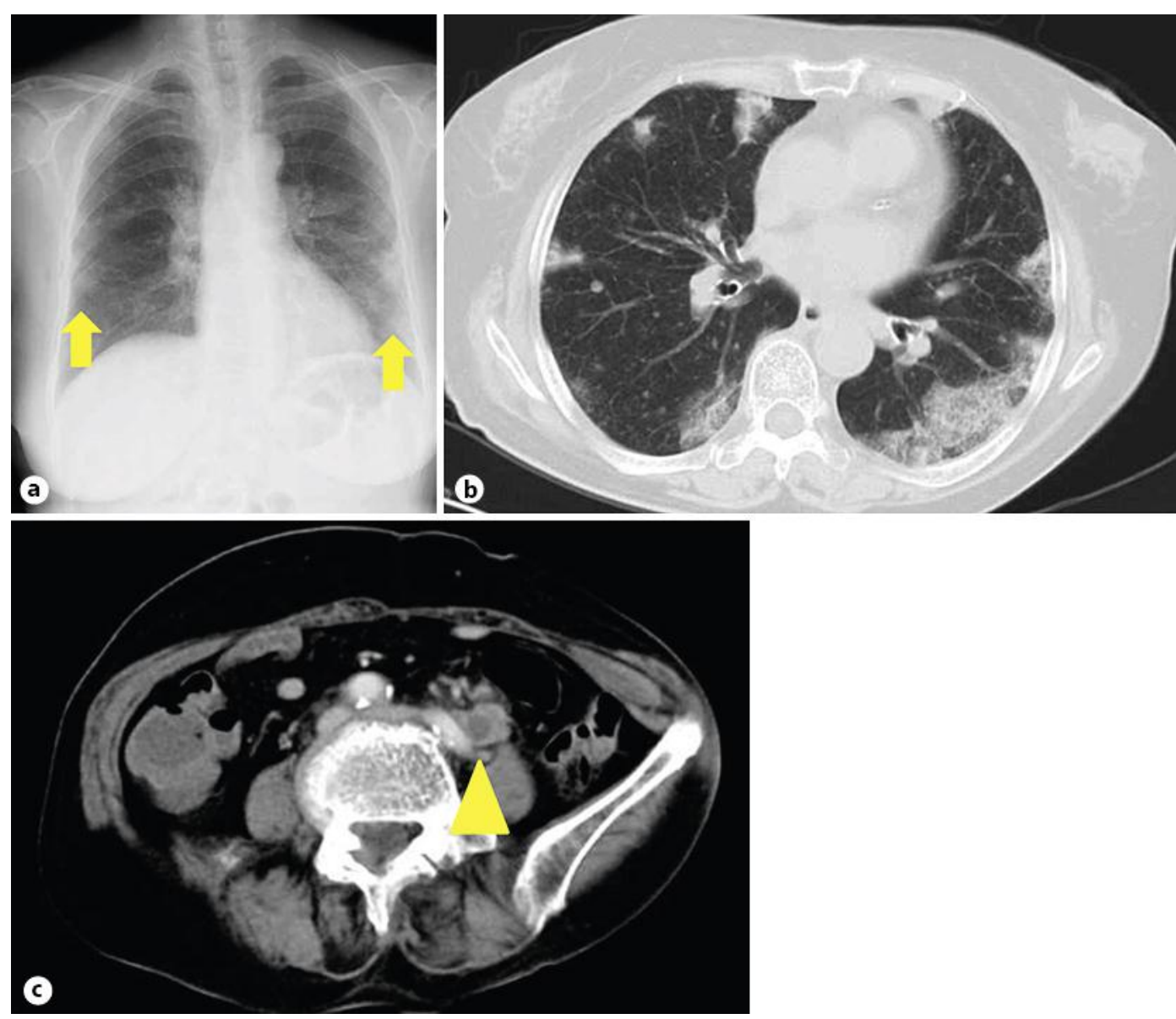

Fig. 1. Radiological findings. a A chest radiograph on admission revealed bilateral opacities (arrows). b A chest HRCT scan on admission revealed GGO of bilateral peripheral lung field predominance. c An enhanced abdominal CT on day 3 of hospitalization revealed a left ureteral nodule involving the left common iliac vein (arrowhead). 


\section{Case Reports in Oncology}

\begin{tabular}{l|l}
\hline Case Rep Oncol 2014;7:605-610 \\
\hline DOI: 10.1159/000366474 & $\begin{array}{l}\text { C 2014 S. Karger AG, Basel } \\
\text { www.karger.com/cro }\end{array}$ \\
\hline
\end{tabular}

Marumo et al.: Pulmonary Tumor Thrombotic Microangiopathy Induced by Ureteral Carcinoma: A Necropsy Case Report

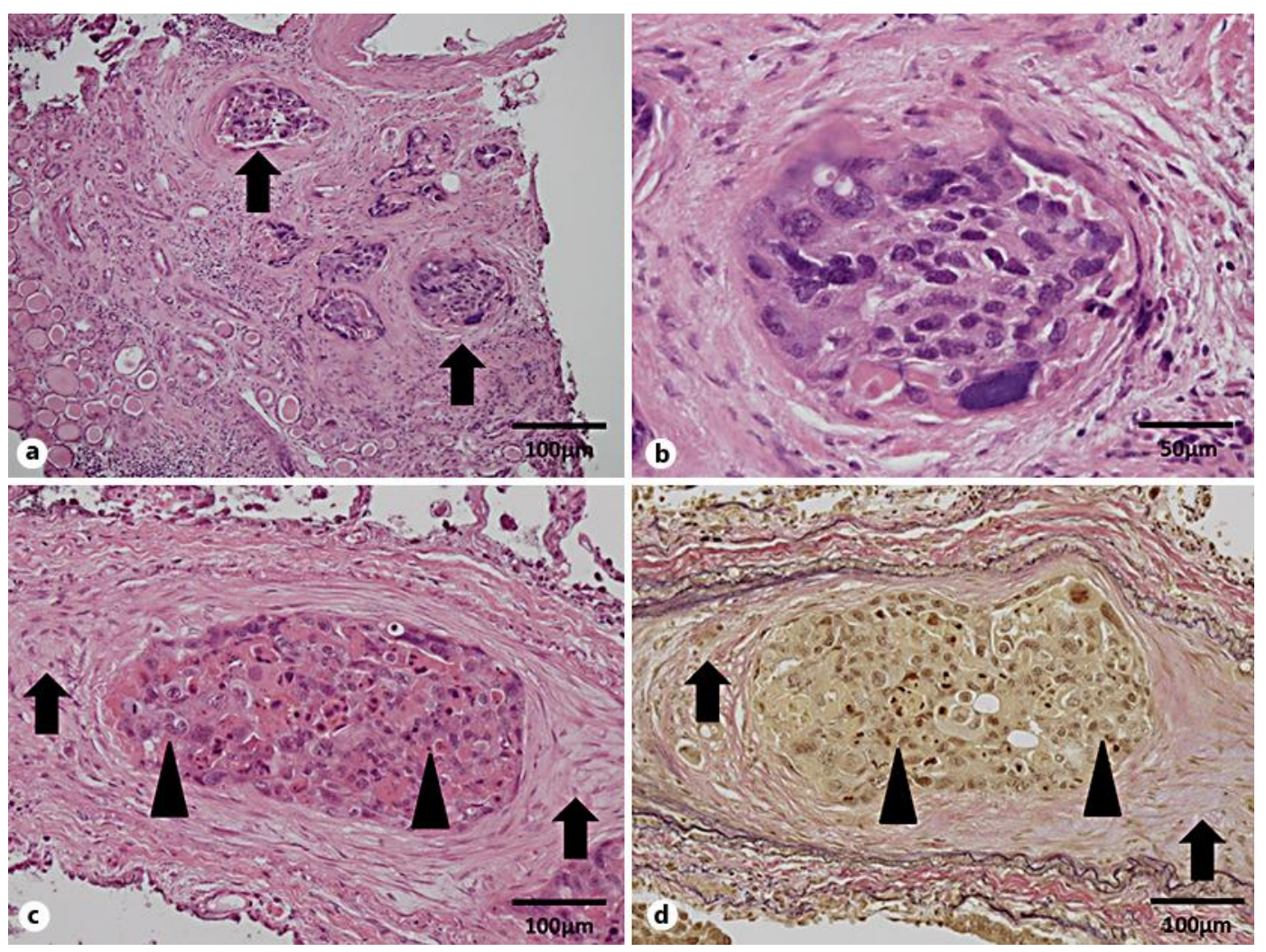

Fig. 2. Pathological findings. Low-power field (a) and high-power field (b) of HE-stained left kidney tissue revealed tumor cells in the ureter (arrows in a). c Lung histology stained with HE. d Lung histology stained with elastic Van Gieson. Tumor cells morphologically similar to the carcinoma of the ureter are present in the pulmonary arterioles (arrows). Marked intimal fibrocellular proliferation and fibrin thrombi are present in the pulmonary arterioles (arrowheads). 